\title{
STRAIGHT-LINE PATH FOLLOWING IN WINDY CONDITIONS
}

\author{
A. Brezoescu*, P. Castillo and R. Lozano \\ Heudiasyc Laboratory, UTC-CNRS 6599, \\ Compiègne, France \\ (cbrezoes, castillo, rlozano)@hds.utc.fr
}

Commission WG I/V

KEY WORDS: UAVs, Flight planning, Flight performance, Wind disturbance, Control law

\begin{abstract}
:
A straight-line following controller for a small and light airplane flying in windy conditions is proposed. In a first time, the lateral dynamics of the plane are derived and the error deviation velocity with respect to the desired trajectory is computed. A simple nonlinear control law is developed in order to impose a linear behavior for the airplane position and to track the desired trajectory. Several simulations, taking into account quasi-constant wind disturbances, are performed to analyze the performance of the closed-loop system. Improved results are obtained including the airplane orientation to counter the wind as an input for the flight planning. In order to validate the proposed control scheme an airplane has been developed based on the classic aerodynamic layout. Future work will introduce the experimental results when applying in real-time the proposed control algorithm.
\end{abstract}

\section{INTRODUCTION}

The Unmanned Aerial Vehicles (UAVs) represent a lightweight solution designed for applications requiring low-altitude surveillance and reconnaissance intelligence. Also, they are best suited to solve dangerous situations and to perform rescue missions since they do not have a human pilot on board. Especially, since it is necessary for UAVs to fly at low altitude in order to observe terrain, UAVs are likely to face a danger to fall down because of irregular wind or obstacles. With advances in flight control and miniaturization of UAVs, they are found to play a key role in urban environments. Most of the mission paths can be defined using a set of way-point and loiters maneuvers, where way-points are straight line segments while loiters are circular orbits. The path following controller has to accurately track the desired path in presence of wind disturbances. In addition, the path following control algorithm must have low computational complexity for use in small and large UAVs without any changes.

Automatic control for airplanes has a long history and these many control techniques are also able to be applied for autonomous UAVs. Since dynamics of airplanes are nonlinear, controllers based on linear theories are not sufficient for trim conditions which are different from the nominal trim condition. In order to overcome this difficulty, it is common to adopt robust and nonlinear control approaches, gain scheduling techniques and so on. For example, in (Rysdyk, 2006) the author develops a guidance law for UAVs to follow straight line, curved trajectories and loiter maneuvers in the presence of winds. Indeed in this paper, the authors introduce a path following approach called "good helmsman" for autonomous monitoring of a target. The approach uses a SerretFrenet formulation to represent the vehicle kinematics in terms of path parameters. The UAV is brought from current path to the desired path by simultaneously regulating the cross track error and course angle error to zero in the Serret-Frenet frame. An observer estimates wind data, which is used to orient path geometry about the target.

A simple path following controller for small UAVs based on the concept of vector fields is presented in (Nelson et al., 2007).

\footnotetext{
${ }^{*}$ Corresponding author
}

The controller generates desired course input in presence of constant wind disturbances and it provides asymptotic following for straight-line and circular paths. A sliding mode controller is used to bring the vehicle to follow the vector field. Another vector field approach is proposed in (Frew and D.Lawrence, 2008). Here, the authors guide the UAV to fly in a circular orbit around a target. The Lyapunov guidance vector field is first designed for a stationary target in the absence of wind, and then a modified version of vector field is applied to the case with a moving target in known constant background wind. Only variable heading rate control input is used to achieve standoff tracking with a constant commanded airspeed. However, the estimation approach for unknown target motion and wind is not taken into account in this paper. Lyapunov vector fields approach was also used for coordinated standoff tracking of stationary or moving targets in UAV formations using a rigid graph theory, see for example (Summers et al., 2009).

In (Zhu et al., 2009) the authors have proposed an adaptive estimation strategy to estimate the velocities of the unknown wind and target motion. In the proposed approach, a variable heading rate controller is designed to achieve standoff tracking of moving target. On the other hand, a guidance law for a micro aerial vehicle, MAV, taking wind into consideration was designed by (Ceccarelli et al., 2007) for the purpose of continuous monitoring of a target in the camera field of view.

In this paper, we study the tracking problem to follow a desired trajectory using an airplane in presence of wind disturbances. We focus mainly in the lateral dynamic of the plane, for this, an analysis of this dynamic is presented in section 2. Likewise, in this section, we introduce the error position and the dynamic velocity of the plane with respect to the desired path. A simple nonlinear control strategy is developed and presented in section 3 in order to follow the trajectory in presence of wind. The validation of the proposed control scheme is done in simulations and the results are depicted in graphs in section 4. Additionally, a prototype of the airplane was constructed to validate the control algorithm, the main characteristics of this prototype are described in section 5. And finally in section 6, the conclusion and future work are discussed. 


\section{PROBLEM STATEMENT}

The most common parameters involved in the UAV control problem are: velocity, altitude, turn rate, flight-path angle, atmospheric turbulence. In this article we deal with the tracking problem of an airplane flying in presence of crosswind. Thus, the mission of the airplane will be to follow a desired trajectory whilst its performance may be modified by the wind. This problem becomes more complex when considering the complete dynamics of the airplane, and in order to simplify analysis and to better understand the problem, hereafter we will focuss to study the lateral dynamics, i.e., the altitude and the pitch and roll angles are quasi-constant (stabilized).

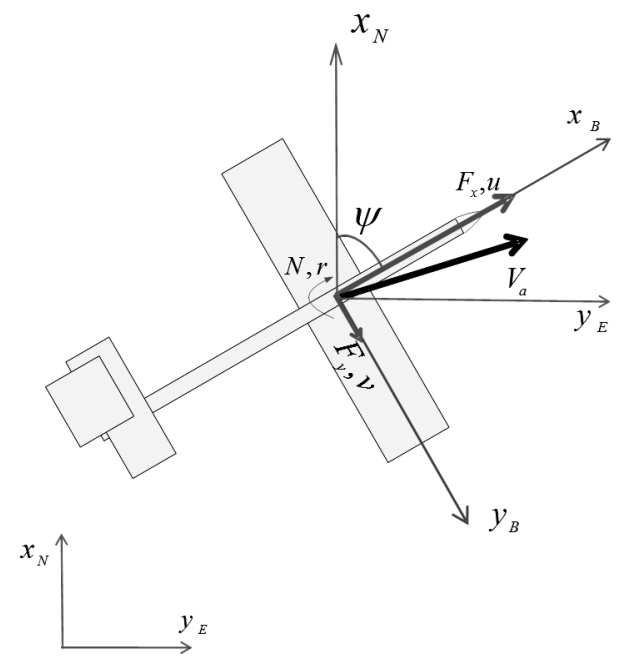

Figure 1: Forces and moments acting on the airplane

Therefore and from Figure 1, the nonlinear dynamic equations for the lateral flight take the form:

$$
\begin{aligned}
\dot{u} & =\frac{X}{m}+r v \\
\dot{v} & =\frac{Y}{m}-r u \\
\dot{x}_{N} & =u \cos \psi-v \sin \psi \\
\dot{y}_{E} & =u \sin \psi+v \cos \psi \\
\dot{r} & =\frac{I_{x x} N}{I_{x x} I_{z z}-I_{x z}^{2}} \\
\dot{\psi} & =r
\end{aligned}
$$

where $u$ and $v$ denotes the airspeed components in the plane $X_{B}$ and $Y_{B}$ respectively, $m$ is the mass of the plane, $\psi$ represents the yaw angle, $r$ describes the yaw rate while $N$ the yawing moment, $X$ and $Y$ are the forces due to aerodynamic effects and to engine thrust, $I_{j j}$ signifies the inertia component in the $j$ axis. The inertial frame is composed by $X_{N}$ and $Y_{E}$ denoting the position in the $x$ axis (North) and in the $y$ axis (East).

Considering a symmetrical airplane with a rigid spinning rotor placed in the front of its body, it can then be considered, without loss of generality, $V_{a}$ acting only in the $x$-axis, see Figure 2 . Then the following expressions can be stated

$$
\begin{aligned}
& u \approx T-D \\
& v<<1
\end{aligned}
$$

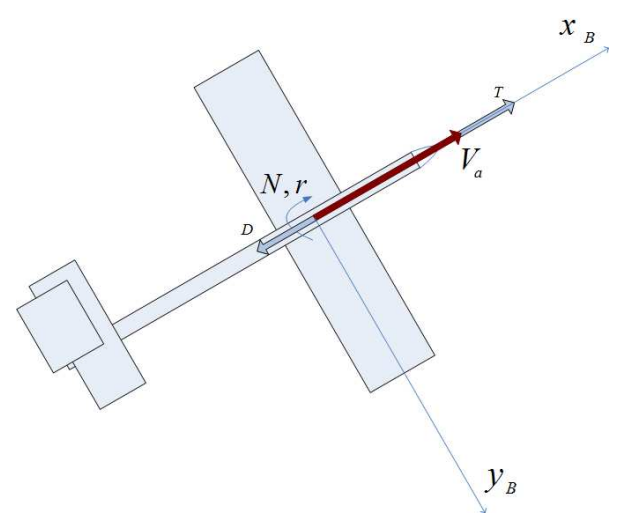

Figure 2: Forces and moments acting on the airplane when considering $v<<1$

where $T$ is the thrust produced by the rotor and $D$ is the drag force. Thus,

$$
\begin{aligned}
& \dot{x}_{N} \approx(T-D) \cos \psi=V_{a} \cos \psi \\
& \dot{y}_{E} \approx(T-D) \sin \psi=V_{a} \sin \psi
\end{aligned}
$$

In real conditions, the plane is generally exposed to wind. If the airplane is experiencing a crosswind, it will be pushed over or yawed away from the wind. Let consider in this study a lateral wind having north and east velocity components, $W_{N}, W_{E}$ respectively, see Figure 3. Hence the velocity in the $c g$ of the aircraft with respect to the air and related in the body fixed frame is given by (Etkin, 1972):

$$
v_{B}^{E}=v_{B}+B_{B}\left[\begin{array}{l}
W_{N} \\
W_{E}
\end{array}\right]
$$

where $v_{B}^{E}=\left[\begin{array}{ll}u^{E} & v^{E}\end{array}\right]^{T}$ is the aircraft speed relative to the airmass in which it is flying expressed in body frame, $v_{B}=\left[\begin{array}{ll}u & v\end{array}\right]^{T}$ describes the airspeed vector in body frame and $B_{B}$ denotes the complete transformation from the inertial frame to the body frame

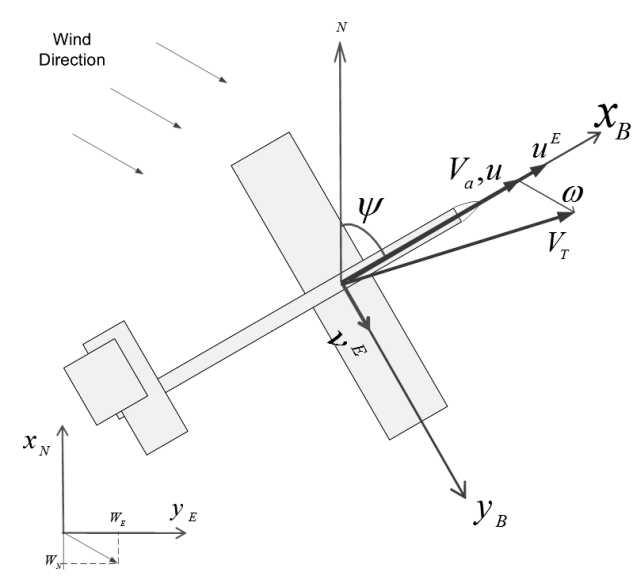

Figure 3: The plane flying with lateral wind. 
assuming zero roll and pitch angles, and it is given by

$$
B_{B}=\left(\begin{array}{ccc}
\cos \psi & \sin \psi & 0 \\
-\sin \psi & \cos \psi & 0 \\
0 & 0 & 1
\end{array}\right)
$$

When equation 9 is added to the nonlinear equations of motion, then $v_{B}^{E}$, rather than $v_{B}$, must be used in the calculation of velocity and orientation of the airplane. To track the flight path relative to Earth we need the velocity components in the directions of the axes of Earth fixed frame. We get these by expressing the velocity vector $v_{B}^{E}$ in Earth frame (Stevens and Lewis, 1992). The differential equations for the coordinates of the flight path are then

$$
\left[\begin{array}{c}
\dot{x_{N}} \\
\dot{y_{E}}
\end{array}\right]=B_{B}^{T} v_{B}^{E}
$$

Therefore, the nonlinear equations representing the airplane movements in the plane $x-y$ and in presence of lateral wind becomes

$$
\begin{aligned}
& \dot{x}_{N}=u^{E} \cos \psi-v^{E} \sin \psi \\
& \dot{y}_{E}=u^{E} \sin \psi+v^{E} \cos \psi
\end{aligned}
$$

with

$$
\begin{aligned}
& u^{E}=u+W_{N} \cos \psi+W_{E} \sin \psi \\
& v^{E}=v-W_{N} \sin \psi+W_{E} \cos \psi
\end{aligned}
$$

Hence, introducing the above into (11) and (12) and using (7) and (8), it follows that

$$
\begin{aligned}
\dot{x}_{N} & =u \cos \psi-v \sin \psi+W_{N} \\
& \approx u \cos \psi+\omega \cos \psi_{\omega} \\
\dot{y}_{E} & =u \sin \psi+v \cos \psi+W_{E} \\
& \approx u \sin \psi+\omega \sin \psi_{\omega}
\end{aligned}
$$

where $W_{N}=\omega \cos \psi_{\omega}, W_{E}=\omega \sin \psi_{\omega}, \omega$ is the wind velocity and $\psi_{\omega}$ describes the wind direction.

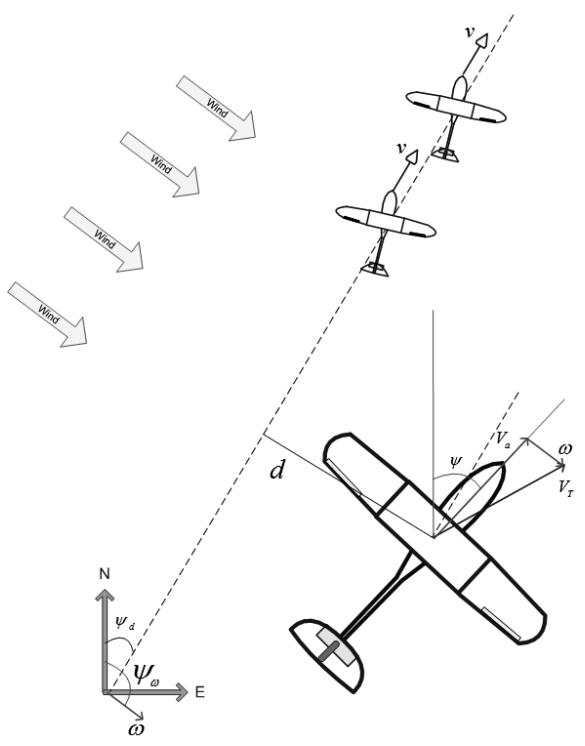

Figure 4: Tracking formulation problem
Remember that the control goal is that the airplane follows a desired trajectory with a constant airspeed $V_{a}$ like in Figure 4. To simplify the analysis, let assume that the desired trajectory is aligned with the North axe of the reference frame, then, the desired path angle, represented in Figure 4 by $\psi_{d}$, is equal to zero. Therefore, the amount of the trajectory deviation will depend on the velocity of the airplane and wind and also on the angle of the wind in relation to the airplane. Thus, the control corrections must be computed in order to reduce the error position, $d$, while controlling the variation of the yaw angle.

Thus, without loss of generality the following equation can be defined:

$$
\dot{d} \equiv \dot{y}_{E}=u \sin (\psi)+\omega \sin \left(\psi_{\omega}\right)
$$

Note that the airplane yaw angle is referred to be the direction that the aircraft nose is pointing while the flight path angle is between the direction of flight and the compass reference (e.g., north), also known as heading.

\section{GUIDANCE LAW DESIGN}

Remember that the crosswind deflects the airplane from its original course. Consequently, it is necessary to point away from the intended course to counteract this effect. Therefore, the proposed guidance law will converge the position error to zero and the yaw angle to the absolute value of the wind correction angle. To this end, the yaw angle will be considered having two components: the component to minimize the position error, $\psi_{e}$, and the component to counter the wind, $\psi_{c}$, i.e.,

$$
\psi=\psi_{e}+\psi_{c}
$$

Consider, in a first time, the airplane aligned with the desired trajectory with the position error very small, thus, $\psi_{e} \approx 0$ and $\dot{d} \approx 0$, then (13) yields

$$
u \sin \left(\psi_{c}\right)+\omega \sin \left(\psi_{\omega}\right)=0
$$

and from the above

$$
\psi_{c}=-\arcsin \left(\frac{\omega \sin \left(\psi_{\omega}\right)}{u}\right)
$$

For simulations purposes, let assume that the wind velocity and the wind orientation are constant. Remember also that the airplane is flying with a constant speed $V_{a} \approx u$ in a plane as shown in Figure 4. Thus, from (13), we obtain

$$
\dot{d}=u \sin (\psi)+k_{\omega}
$$

where $k_{\omega}$ is constant, see (13) and (15).

Notice that the above equation is relatively proportional to the variation of the yaw angle and it can be controlled using the rudder of the airplane. The rudder of the plane can be operated using the servo motor angular acceleration. Then, taking the second and third derivate with respect to the time from (16) and using (5) and (6), it follows that

$$
\begin{aligned}
\dddot{d} & =u\left(\ddot{\psi} \cos (\psi)-\dot{\psi}^{2} \sin (\psi)\right) \\
& =u\left(J N \cos (\psi)-\dot{\psi^{2}} \sin (\psi)\right.
\end{aligned}
$$

with $J=\frac{I_{x x}}{I_{x x} I_{z z}-I_{x z}^{2}}$. 
Proposing

$$
J N=\dot{\psi}^{2} \tan \psi-3 \dot{\psi}-\frac{3 \dot{d}+d}{u \cos \psi}
$$

then, the closed-loop system becomes

$$
\dddot{d}=-3 \ddot{d}-3 \dot{d}-d
$$

or $s^{3}+3 s^{2}+3 s+1=0$, that represents a stable polynomial. Then from the above, it follows that $d^{(i)} \rightarrow 0$ for $i=0,1,2,3$.

\section{SIMULATION RESULTS}

A previous analysis of the nonlinear model is presented for different conditions (with and without wind). Figure 5 introduces the behavior of the plane without wind whilst Figure 6 illustrates the states affected by the wind. In this case we consider a crosswind having North and East velocity components of $W_{N}=$ $1 \mathrm{~m} / \mathrm{s}, W_{E}=3 \mathrm{~m} / \mathrm{s}$ respectively. The values parameters are shown in Table 1. Notice from Figure 6 that the body-axis components of inertial velocity and the sideslip angle are presented.
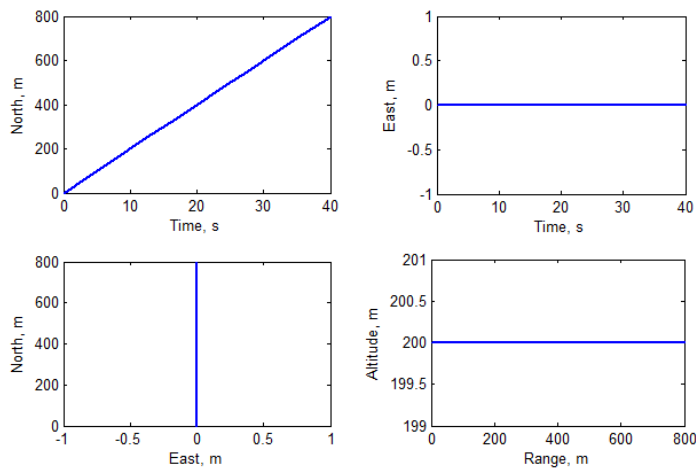

Figure 5: Earth-Relative Aircraft Location

\begin{tabular}{|c|c|}
\hline Meaning & Value \\
\hline Airplane Velocity & $20 \mathrm{~m} / \mathrm{s}$ \\
Airplane Orientation & $0^{\circ}$ \\
Wind Velocity & $0 \mathrm{~m} / \mathrm{s}$ \\
Altitude & $200 \mathrm{~m}$ \\
\hline
\end{tabular}

Table 1: Flying parameters without wind
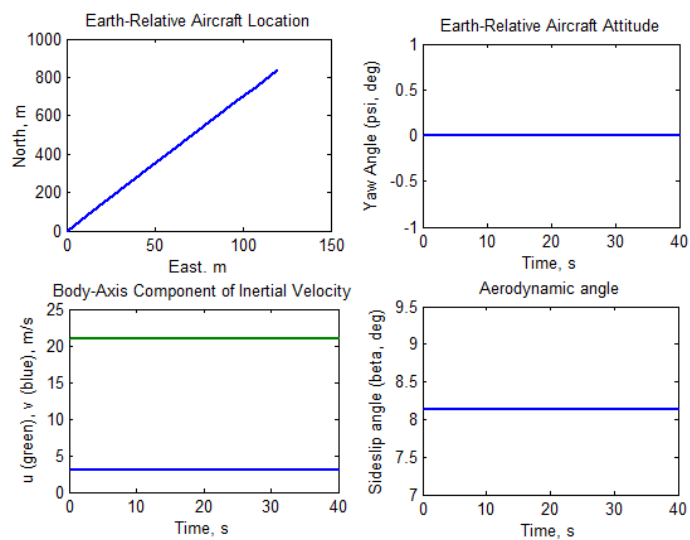

Figure 6: Airplane states in presence of wind
The proposed control strategy is validated in closed-loop system in simulations with various wind conditions. The UAV airspeed is considered $20 \mathrm{~m} / \mathrm{s}$ while the wind velocity is $14 \mathrm{~m} / \mathrm{s}$. There is no initial path deviation and the wind direction is North-East perpendicular to the desired path, see Figure 7. The resulting trajectories in figures are plotted with blue line for Wind Correction Controller (WCC) and red line for No Wind Correction Controller (NWCC).

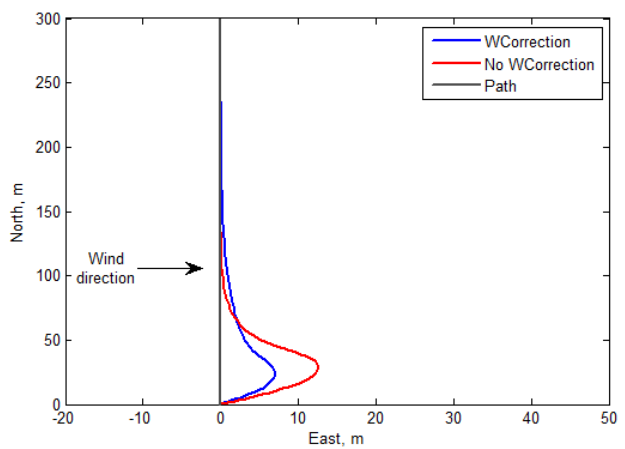

Figure 7: Airplane response in presence of wind

Notice also from Figure 7 that the error using the WCC is $7.5 \mathrm{~m}$ while employing the NWCC this error is highly affected by crosswind diverting almost $13 \mathrm{~m}$ from the path. In order to compare the performance of the two controllers we vary the wind speed magnitude and the wind orientation. In Figure 8, we illustrate the state responses for wind values of $1 \mathrm{~m} / \mathrm{s}, 6 \mathrm{~m} / \mathrm{s}$ and $11 \mathrm{~m} / \mathrm{s}$ while the wind blows perpendicular to the desired path. On the other hand, changing the wind orientation while keeping a constant wind velocity of $11 \mathrm{~m} / \mathrm{s}$, also results in a perturbed system, see Figure 9.

For comparative studies we plot, in Figures 10 and 11, the results obtained employing the NWCC. The controller is affected by crosswind since it deviates more from the desired trajectory. The different wind magnitudes used to simulate the performance of the controller are $1 \mathrm{~m} / \mathrm{s}, 6 \mathrm{~m} / \mathrm{s}$ and $11 \mathrm{~m} / \mathrm{s}$ respectively. Observe in these figures that the controller shows good performance when considering the airplane orientation to counter the wind.

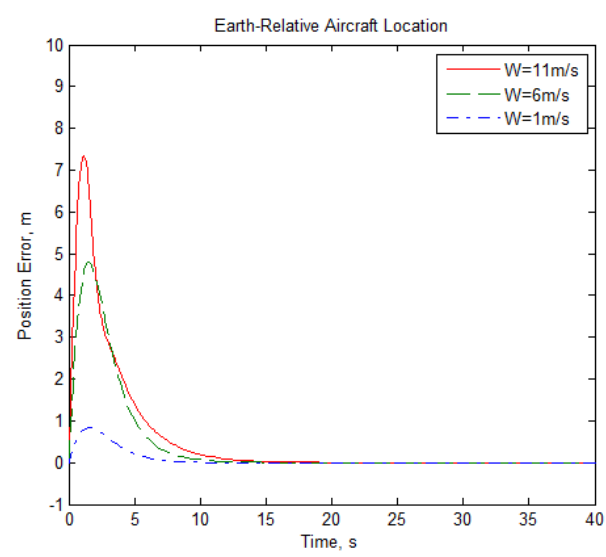

Figure 8: Position error when employing the Wind Correction Controller - WCC 


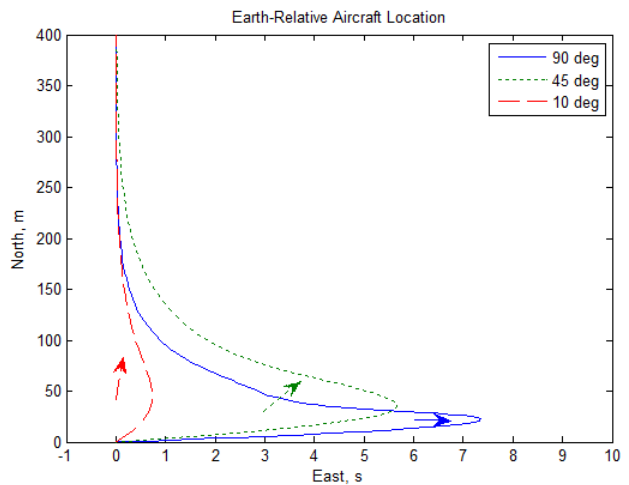

Figure 9: Earth relative position for different wind orientation

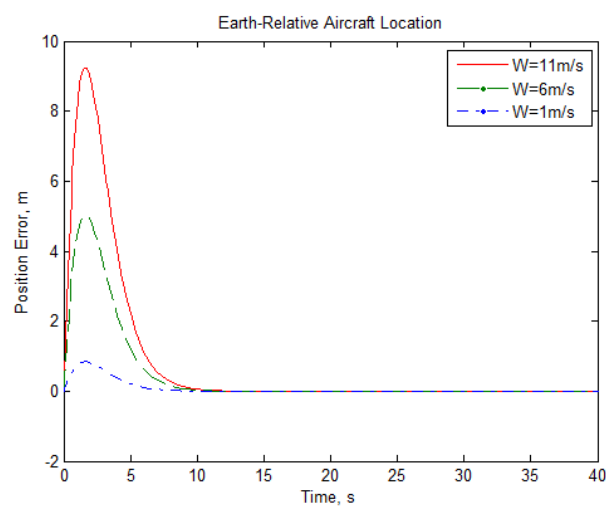

Figure 10: Position error reponse when using the No Wind Correction Controller - NWCC

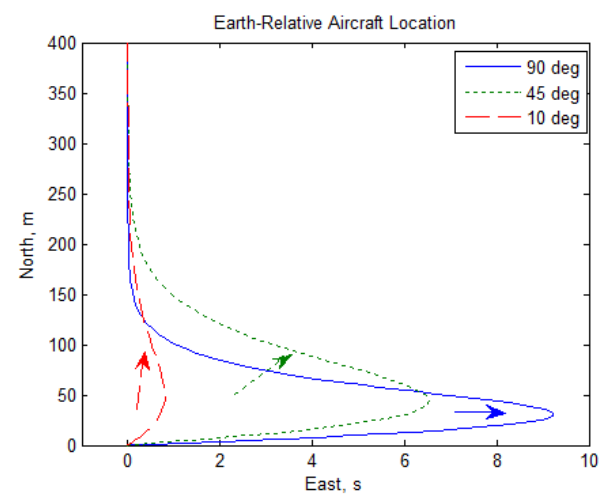

Figure 11: Earth relative position for different wind orientation

For the following simulations we assumed that the wind is characterized by constant velocity but it increases its speed during the flight. The wind velocity is plotted in Figure 12. If we have the knowledge about the wind parameters, we can compute the wind correction angle and the airplane will remain closer to the desired path. The positions errors are plotted in Figure 13 and 14 for different wind conditions. The parameters values used in this simulations are summarized in Table 2.

Once more we can notice a better performance of the controller

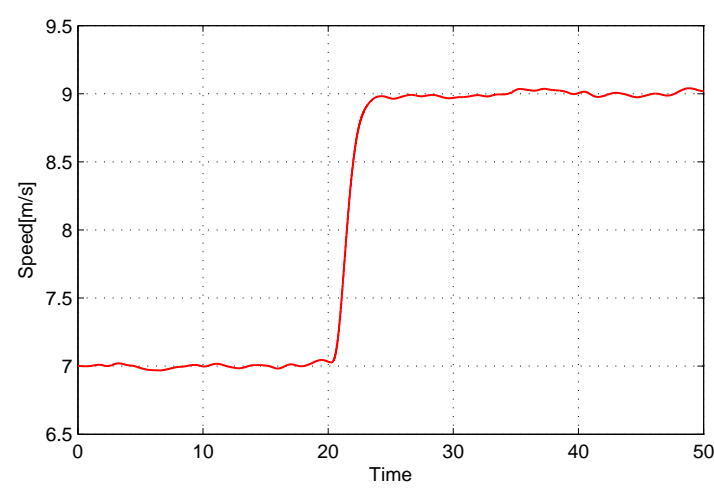

Figure 12: Wind velocity

\begin{tabular}{|c|c|c|}
\hline Symbol & Meaning & Value \\
\hline$v$ & Airplane Velocity & $20 \mathrm{~m} / \mathrm{s}$ \\
$d_{0}$ & Initial Error & $2 \mathrm{~m}$ \\
$\omega$ & Wind Velocity & $7 \mathrm{~m} / \mathrm{s}$ \\
$\psi_{\omega}$ & Wind Orientation & $0^{\circ}, 45^{\circ}, 90^{\circ}, 180^{\circ}$ \\
\hline
\end{tabular}

Table 2: Control parameters

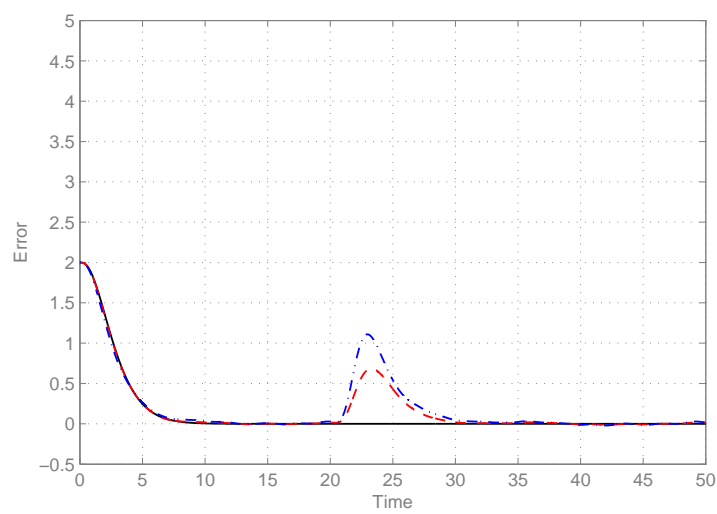

Figure 13: Position error using WKC with different conditions of wind orientation: $\psi_{\omega}=0^{\circ}, \psi_{\omega}=30^{\circ}, \psi_{\omega}=90^{\circ}$

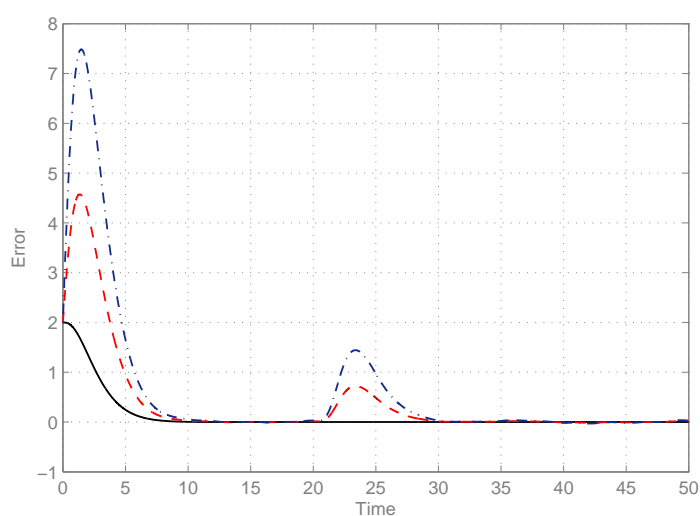

Figure 14: Position error using NWKC with different conditions of wind orientation: $\psi_{\omega}=0^{\circ}, \psi_{\omega}=30^{\circ}, \psi_{\omega}=90^{\circ}$

when computing the airplane orientation to resist to the wind as input for the flight planning. The WCC controller allows the airplane to stay close to the path as long as the wind does not change its values. The path deviation remains small even for small variations of wind parameters. 


\section{AIRPLANE CONFIGURATION}

In this section we introduce briefly the airplane which we developed in order to validate the proposed control law. Its configuration is based on the classic aerodynamic layout and it is built of polystyrene foam sheet and carbon fiber tubes. The airplane is powered by a brushless motor placed in front of the body. The airplane has five wings, a main airfoil-shaped wing fixed to the body, a couple of ailerons, an elevator and a rudder. Servo motors are attached to ailerons, the elevator and the rudder as control surface actuators. Parameters of this airplane are given in Table 3 and a photo of the airplane is presented in Figure 15.

\begin{tabular}{|l|c|}
\hline Parameter & Value \\
\hline Airfoil & Shape NACA \\
Wing chord $(\bar{c})$ & $0.23 \mathrm{~m}-0.19 \mathrm{~m}$ \\
Wing span (b) & $1.4 \mathrm{~m}$ \\
Aspect ratio (AR) & 6.49 \\
Wing Area (s) & $0,302 \mathrm{~m}^{2}$ \\
Mass Vehicle $(\mathrm{kg})$ & $0.70 \mathrm{~kg}$ \\
Length $(\mathrm{m})$ & $1 \mathrm{~m}$ \\
\hline
\end{tabular}

Table 3: Parameters of the airplane.

The airplane is able to take off from a runway and to land on the ground. The on-board computer system is the RabbitCore RCM4300 Microprocessor and it is connected to an airspeed and altitude sensor, and a Futaba system for a servo signal generator/receiver unit. Sensor unit consists of three accelerometers, three gyroscopes and a magnetometer. All servo motors are controlled manually via radio control in manual mode, or automatically by an on-board computer system in auto mode.

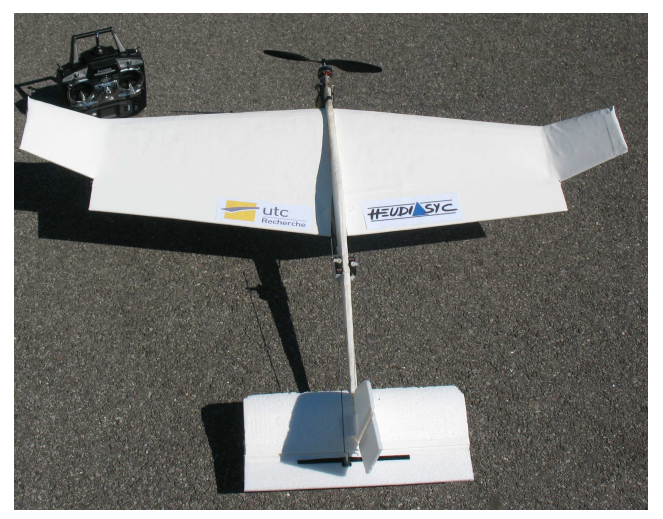

Figure 15: The airplane prototype.

\section{CONCLUSION}

In this paper, a trajectory following controller for a small and light airplane flying in windy conditions was proposed. In order to achieve it, the lateral dynamic of the vehicle and the equation of the position deviation velocity with respect to the desired path were derived.

Some hypothesis have been stated in order to simplify the problem and the analysis and a simple control law was proposed to tracking a desired trajectory. In addition, the proposed control law imposes a linear behavior for the airplane position and it has shown a good performance in simulations.

Improved results have been obtained including the airplane orientation to counter the wind as input for the flight. Therefore, the position error remains small for small variations of wind parameters.

The simulation results justify the reliability and efficiency of the proposed control law even considering the wind velocity variable. An airplane has been developed based on the classic aerodynamic layout having a unibody fuselage with wings providing majority of the lift. Future work will be done validating the proposed control strategy in the prototype.

\section{REFERENCES}

Ceccarelli, N., Enright, J. J., Frazzoli, E., Rasmussen, S. J. and Schumacher, C. J., 2007. Micro uav path planning for reconnaissance in wind. American Control Conference pp. 5310-5315.

Etkin, B., 1972. Dynamics of atmospheric flight.

Frew, E. and D.Lawrence, 2008. Cooperative standoff tracking of moving targets using lyapunov guidance vector fields. AIAA Journal of Guidance, Control, and Dynamics 31(2), pp. 290-306.

Nelson, D. R., Barber, D. B., McLain, T. W. and Beard, R. W., 2007. Vector field path following for small unmanned air vehicles. IEEE Transactions on Robotics and Automation 23(3), pp. 519-529.

Rysdyk, R., 2006. Unmanned aerial vehicle path following for target observation in wind. Journal of Guidance, Control, and Dynamics 29(5), pp. 1092-1100.

Stevens, B. L. and Lewis, F. L., 1992. Aircraft control and simulation.

Summers, T. H., Akella, M. R., and Mears, M. J., 2009. Coordinated standoff tracking of moving targets: Control laws and information architectures. Journal of Guidance, Control, and Dynamics 32(1), pp. 56-69.

Zhu, S., Wang, D. and Chen, Q., 2009. Standoff tracking control of moving target in unknown wind. 48th IEEE Conference on Decision and Control. 